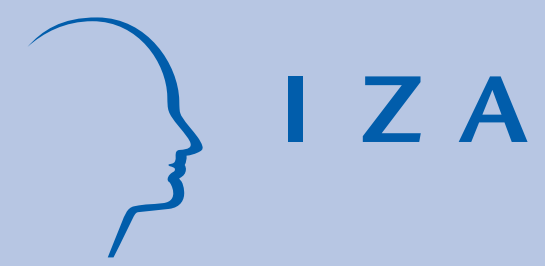

IZA Standpunkte Nr. 85

Digitalisierung und Arbeitsmarkt:

Aktuelle Entwicklungen und sozialpolitische Herausforderungen

Werner Eichhorst

Holger Hinte

Ulf Rinne

Verena Tobsch

Juli 2016 


\title{
Digitalisierung und Arbeitsmarkt: Aktuelle Entwicklungen und sozialpolitische Herausforderungen
}

\author{
Werner Eichhorst \\ IZA \\ Holger Hinte \\ $I Z A$ \\ Ulf Rinne \\ IZA \\ Verena Tobsch \\ INES Berlin
}

IZA Standpunkte Nr. 85

Juli 2016

IZA

Postfach 7240

53072 Bonn

Tel.: (0228) 3894-0

Fax: (0228) 3894-180

E-Mail: iza@iza.org

Die Schriftenreihe „IZA Standpunkte” veröffentlicht politikrelevante Forschungsarbeiten und Diskussionsbeiträge von IZA-Wissenschaftlern, Fellows und Affiliates in deutscher Sprache. Die Autoren sind für den Inhalt der publizierten Arbeiten verantwortlich. Im Interesse einer einheitlichen Textzirkulation werden Aktualisierungen einmal publizierter Arbeiten nicht an dieser Stelle vorgenommen, sondern sind gegebenenfalls nur über die Autoren selbst erhältlich.

Das IZA ist ein privates, unabhängiges Wirtschaftsforschungsinstitut, das als gemeinnützige $\mathrm{GmbH}$ durch die Deutsche Post-Stiftung gefördert wird. Zentrale Tätigkeitsfelder sind die intensive Forschungstätigkeit auf allen Gebieten der Arbeitsökonomie und die darauf gründende evidenzbasierte Politikberatung zu Arbeitsmarktfragen. Die Mitglieder des weltweiten IZA-Forschernetzwerks sind den „IZA Guiding Principles of Research Integrity“ verpflichtet. 
IZA Standpunkte Nr. 85

Juli 2016

\section{ZUSAMMENFASSUNG}

\section{Digitalisierung und Arbeitsmarkt: Aktuelle Entwicklungen und sozialpolitische Herausforderungen}

Der vorliegende Beitrag setzt sich mit den Auswirkungen der fortschreitenden Digitalisierung auf unsere Arbeitswelt auseinander. Nach einer Diskussion möglicher Folgen dokumentiert eine empirische Bestandsaufnahme den bereits einsetzenden Wandel der Berufe und Erwerbsformen sowie die Rolle der Plattform-Ökonomie (u.a. mit dem Phänomen der „SoloSelbständigkeit"). Aus diesen Überlegungen werden aktuelle und künftige sozialpolitische Herausforderungen abgeleitet und Lösungsansätze diskutiert, um den Wandel gesellschaftlich fair zu gestalten.

JEL-Codes: J08, J24, O33, O38

Schlagworte: Digitalisierung, Roboter, Automatisierung, Zukunft der Arbeit, Industrie 4.0, technischer Wandel, Sharing Economy, Plattform-Ökonomie, Arbeitsmarkt, Sozialstaat

Kontaktadresse:

Werner Eichhorst

Institut zur Zukunft der Arbeit (IZA)

Schaumburg-Lippe-Straße 5-9

53113 Bonn

Deutschland

E-mail: eichhorst@iza.org 


\section{Einleitung}

Die fortschreitende Digitalisierung von Prozessen und Arbeitsabläufen beeinflusst bereits heute unser tägliches Handeln - so etwa die Art unserer Kommunikation und unseres sozialen Umgangs, insbesondere aber auch die Funktionsweise unseres Wirtschaftens und Arbeitens. Wie wird dies erst künftig sein? Spekuliert und diskutiert wird in diesem Zusammenhang über zahlreiche mögliche, kurz- wie langfristige Folgen. Ein zentraler Aspekt sind wachsende Ungleichheiten auf dem Arbeitsmarkt, welche sich unter anderem in der Lohnspreizung sowohl am unteren als auch am oberen Rand der Lohnverteilung ausdrücken - aber auch in der Dualisierung des Arbeitsmarktes via atypischer oder „prekärer“ Beschäftigung, in der Ersetzbarkeit menschlicher Arbeit durch Roboter und Maschinen sowie in neu entstehenden Arbeitsformen in der sogenannten Plattform-Ökonomie.

In der einschlägigen Literatur werden hauptsächlich vier Erklärungsfaktoren für die Zunahme der (wahrgenommenen oder tatsächlichen) Unsicherheiten und Ungleichheiten in der Arbeitswelt angeführt. Neben dem technischen Fortschritt mit den Phänomenen der Digitalisierung und Automatisierung sind dies vor allem die Globalisierung mit den Tendenzen zum Outsourcing und Offshoring (vgl. u.a. Autor et al., 2016), die Flexibilisierung bzw. Deregulierung mit einem Rückgang der Tarifbindung und einer Liberalisierung flexibler Erwerbsformen (vgl. u.a. Eichhorst/Tobsch, 2015) sowie das Aufkeimen zahlreicher neuer Internetplattformen, die virtuelle Marktplätze von Privatanbietern oder Gruppen erschaffen und so Ausgangspunkte für ein neuartiges Angebot von Dienstleistungen aller Art sind, das Marktlücken ausfüllt und so auch die Nachfrage stimuliert (vgl. u.a. Eichhorst/Spermann, 2015). Obgleich unser Augenmerk im Folgenden in erster Linie auf dem Zusammenspiel von Digitalisierung und Erwerbsformen liegt, sollte beachtet werden, dass die oben genannten Faktoren in Wechselbeziehungen zueinander stehen und dass sich ihre Folgen in der Regel gegenseitig verstärken.

Die öffentliche Debatte bewegte sich jüngst sehr stark im Umfeld möglicher Negativfolgen des technischen Fortschritts, etwa in Form einer zunehmenden sozialen Ungleichheit, eines massenhaften Verlustes von automatisierbaren Arbeitsplätzen oder einer wachsenden Dominanz von Kapitaleinkommen gegenüber den Erlösen aus menschlicher Arbeit. In diesem Zusammenhang stellt die Automatisierung nicht zuletzt auch die Frage nach der Kapital- und Gewinnbeteiligung von Beschäftigten völlig neu (vgl. Freeman, 2015). Durch die weitere Integration industrieller Produktion und neuester Kommunikationstechnologien im Zuge einer „Industrie 4.0“ (vgl. BMWi, 2015; Hirsch-Kreinsen, 2016) gewinnen die bereits heute zu beobachtenden Veränderungen für fast jeden Beschäftigten eine noch größere Relevanz. 
Obwohl davon ausgegangen werden kann, dass die rapide Digitalisierung verschiedener Arbeitsbereiche eine bedeutende strukturelle Veränderung der Beschäftigungsmöglichkeiten zur Folge haben wird (vgl. Brynjolfsson/McAfee, 2011), erscheint es ratsam, insgesamt Vorsicht bei der Einschätzung der Geschwindigkeit von signifikanten Änderungen in der Arbeitswelt walten zu lassen (vgl. Eichhorst et al., 2015). Bereits Anfang des 19. Jahrhunderts sorgten sich englische Textilarbeiter („Ludditen“) um die Auswirkungen der industriellen Revolution, was in der gezielten Zerstörung von Maschinen gipfelte (vgl. Hobsbawm, 1952). Auch Keynes warnte in den 1930er Jahren vor "technologischer Arbeitslosigkeit", die sich infolge des beschleunigten technischen Fortschritts weit verbreiten werde (vgl. Keynes, 1930). Und vor rund 20 Jahren war die Vorhersage einer raschen Dominanz der digitalen Welt so populär, dass bereits von einem „Ende der Arbeit“ gesprochen wurde (vgl. Rifkin, 1995) - ohne dass sich dies in der Folge bewahrheitet hätte.

Die bisherigen Entwicklungen im Bereich der Informations- und Kommunikationstechnik (IKT) haben jedoch bereits heute eine enorme Bedeutung für das tägliche soziale und berufliche Leben und lassen weitere substanzielle Veränderungen erwarten. Eine viel beachtete Studie unterstellt beispielsweise, dass rund $47 \%$ der gesamten Beschäftigung in den Vereinigten Staaten zumindest mittelfristig (d.h. in den nächsten 10 bis 20 Jahren) davon bedroht ist, durch Maschinen, Roboter und Computerprogramme ersetzt zu werden (vgl. Frey/Osborne, 2013). Andere Untersuchungen deuten allerdings eher darauf hin, dass weder der technologische Fortschritt im Allgemeinen, noch die Digitalisierung im Speziellen menschliche Arbeitskraft in massivem Umfang ersetzen werden, sondern - wie in anderen Epochen auch die nicht mehr zeitgemäßen Arbeitsformen durch neue ersetzt werden (vgl. u.a. Eichhorst et al., 2013; Eichhorst/Buhlmann, 2015; Rinne/Zimmermann, 2016).

Die vor uns liegende, noch nicht in allen Aspekten genau abschätzbare technologische Neuorganisation der Arbeitswelt wird in jedem Fall erhebliche Auswirkungen auf Produktionsfaktoren, Berufe und Erwerbsformen haben. Im Ergebnis entstehen neue Risiken für einzelne Arbeitsmarktsegmente, aber es eröffnen sich auch vielfältige Chancen und Potenziale, die im günstigsten Fall sogar für eine Überkompensation der wegfallenden Arbeitsplätze sorgen könnten. Allein für Deutschland wird das Wachstumspotential durch Industrie 4.0 in einem positiven Szenario bis zum Jahr 2025 auf 425 Milliarden Euro geschätzt (vgl. Roland Berger, 2015). Es stellt sich in diesem Zusammenhang jedoch die Frage, inwieweit diese mutmaßlich erzielbaren Wohlfahrtsgewinne ohne politische Maßnahmen (z.B. zur Stärkung der Mitarbeiterbeteiligung) zu ungleich verteilt werden und einzelne Gruppen einem erhöhten Risiko ausgesetzt sein werden, Leidtragende einer immer stärkeren Polarisierung des Arbeitsmarktes zu sein. Mit anderen Worten: Über die Gestaltung der digitalen Arbeitswelt hinaus werden wir wohl vor erheblichen sozialpolitischen Herausforderungen 
stehen, um die gesellschaftliche Chancengleichheit ebenso wie die steigenden Beschäftigungsrisiken bestmöglich abzusichern.

Der vorliegende Betrag skizziert zunächst die Dynamik der Digitalisierungsprozesse und nimmt eine empirische Bestandsaufnahme zum Wandel der Erwerbsarbeit im Verlauf der letzten beiden Jahrzehnte vor. Dabei wird auch die Rolle der neuen Plattform-Ökonomie diskutiert. Im Anschluss werden die zentralen sozialpolitischen Handlungsfelder benannt.

\section{Mögliche Auswirkungen der Digitalisierung}

Die Digitalisierung der Arbeitswelt lässt sich als ein tiefgreifender Prozess der Durchdringung von Wertschöpfungs- und Dienstleistungsprozessen durch vor allem internetbasierte Technologien begreifen. Er führt unter anderem dazu, dass sich ganze Arbeitsfelder in Teilmodule zerlegen, automatisieren und flexibel miteinander vernetzen lassen. Auf diese Weise können sowohl lokale als auch weltumspannende Produktionsketten leichter zueinander kompatibel gemacht und somit weit effizienter organisiert werden; Routinen lassen sich in die Hand intelligenter Maschinen legen und auf diese Weise optimieren. An anderer Stelle schafft die Digitalisierung anspruchsvolle Aufgabenfelder der Prozesskontrolle, Koordination und kreativen Gestaltung. ${ }^{1}$

Über die möglichen Auswirkungen der Digitalisierung mit Blick auf die Beschäftigung herrscht noch erhebliche Ungewissheit. Es gibt international sowohl empirische Belege für die Annahme, der flächendeckende Einsatz von Robotern führe zu einer Aufwertung aller Qualifikationsstufen („Upgrading“) als auch für die These, das mittlere Qualifikationssegment werde zum eigentlichen Opfer der digitalen Arbeitswelt, indem es auf das Niveau einfacher Tätigkeiten herabgedrängt werde, während hohe Qualifikationsebenen in noch stärkerem Maße die Gewinner darstellten (Polarisierung; vgl. Rinne/Zimmermann, 2016).

Bei näherer Betrachtung - die im nachfolgenden Abschnitt noch vertieft werden soll erscheint es tatsächlich eher naheliegend, dass vor allem mittlere Qualifikationsstufen von der Digitalisierung negativ betroffen sein werden, als dass angesichts der ohnehin schon sehr weitreichenden Rationalisierung einfacher Tätigkeiten nochmals geringer qualifizierte Arbeitsplätze in massivem Umfang wegbrechen werden. Dessen ungeachtet stellt der Prozess der Digitalisierung insbesondere die Weiterbildungswirtschaft vor eine immense Herausforderung. Denn das „Upgrading“ von Qualifikationen - hier gemeint die Befähigung zum Einsatz komplexer Technologien - setzt die entsprechenden betrieblichen wie außerbetrieblichen Qualifizierungsangebote voraus. Dabei kann sich die Branche allerdings

\footnotetext{
${ }^{1}$ Brynjolfsson/McAffee (2014) benennen drei maßgebliche Merkmale des technischen Fortschritts in der Ära digitaler Hardware, Software und Netzwerke: Er sei „exponentiell, digital und kombinatorisch“.
} 
just auch die Vorteile der Digitalisierung zunutze machen. Smartphone- und Tablet-basierte Weiterbildungsmodule können einen wertvollen Beitrag zur Organisation des dringend erforderlichen kontinuierlichen Lernens leisten.

In jedem Fall werden mit der Digitalisierung auch Trends auf dem Arbeitsmarkt ihre beschleunigte Fortsetzung finden, die bereits für die jüngere Vergangenheit prägend waren. Gemeint ist hier vor allem die Flexibilisierung in Form von individuell zugeschnittenen Werkoder Zeitarbeitsverträgen, Solo-Selbstständigkeit und multiplen Jobs. Im Verlauf der Digitalisierung neu entstehende Arbeitsplätze dürften mit nur unter bestimmten Voraussetzungen als klassische Normalarbeitsverhältnisse mit langfristiger Beschäftigungsperspektive, tarifvertraglicher Eingruppierung und voller sozialer Absicherung konzipiert werden. Denn die Unternehmen werden durch den Einsatz neuer Technologien leichter denn je in die Lage versetzt, Arbeitsmodule bei Bedarf auch kurzfristig extern zuzukaufen, generell stärker in Kategorien von befristeter Projektarbeit in temporär agierenden Netzwerken zu denken und ihre Kernbelegschaft in denjenigen Segmenten, wo kein Fachkräftemangel herrscht, auf ein gewünschtes Mindestmaß zu konzentrieren.

Die digital ermöglichte Zergliederung von Aufgaben und Tätigkeitsprofilen schafft zugleich einen unmittelbaren Anreiz zum Aufbau einer dezentralen Plattform-Ökonomie: Einzelanbieter offerieren ihre Dienstleistungen im Rahmen eines professionell organisierten Internet-Portals und erzeugen auf diese Weise einen intensiven Wettbewerb. Die Vermittlung der angebotenen Dienstleistungen erfolgt online und über Smartphone-Apps, kann also jederzeit nahezu barrierefrei erfolgen. Angebote wie Uber als Vermittler für Fahrdienstleistungen oder auch TaskRabbit oder Helpling als Vermittler für (nachbarschaftliche) Dienstleistungen aller Art und handwerkliche Tätigkeiten zeigen, wie schnell und wie erfolgreich solche Geschäftsmodelle ihren Aufstieg nehmen können. Ein weiteres Beispiel ist Airbnb als virtueller Marktplatz für die Buchung und Vermietung von Unterkünften. Die Auswirkungen dieses neuen Angebotes spüren dann nicht nur Wettbewerber in der Hotelbranche, sondern Effekte zeigen sich auch im Immobilienmarkt.

Die Plattform-Ökonomie findet ihre Grenze also keineswegs bei einfachen Dienstleistungen. Hochwertige Beratungstätigkeiten und Arbeitsmodule von hochgradig spezialisierten Experten zählen prinzipiell ebenso zum Katalog der Möglichkeiten. Deshalb ist auch keineswegs bereits ausgemacht, dass die digitale Arbeitswelt von morgen verstärkt "Lohndumping" betreiben wird und die Unternehmen nur noch Niedriglöhne für gute Arbeit anzubieten bereit sein werden. Im Gegenteil: Es dürften sich sehr bald Fragen wie diejenige nach einer systematischen Mitarbeiter-Kapitalbeteiligung anders und drängender stellen als je zuvor. "Who owns the robots rules the world" (Freeman, 2015) - ist es dann nicht nur 
folgerichtig, wenn die Beschäftigten in und um das Unternehmen herum vom Einsatz der Roboter auch durch innovative Kapitalbeteiligungsmodelle profitieren?

Es liegt auf der Hand, dass all dies unmittelbare Konsequenzen auch für die Reichweite und Tragfähigkeit von Tarifverträgen haben wird. Ebenso leuchtet ein, wie sehr solche digital unterstützten Arbeitsformen den gewerkschaftlichen Organisationsgrad in der Industrie unterminieren könnten (vgl. Eichhorst et al., 2015). Nicht zuletzt wird an dieser Stelle deutlich, dass sich damit Fragen der sozialen Absicherung ganz neu stellen. Es ist nicht unwahrscheinlich, dass am "Ende“ des Digitalisierungsprozesses auch eine neue „(Sozial-)Versicherungslandschaft 4.0“ entstanden sein wird, die elementare Merkmale einer Bürgerversicherung aufweist und jede Beschäftigungsform - vom Selbstständigen und Beamten bis hin zum Plattform-Unternehmer in eigener Sache - einschließt. Dieser Schritt erscheint naheliegend, wenn sich die Grenzen zwischen den Erwerbsformen zunehmend auflösen. Für viele Erwerbstätige sind die traditionellen Klassifikationen als „abhängig Beschäftigte“ oder „Selbstständige“ schon heute nicht mehr richtig passend. Abschnitt 4 geht hierauf näher ein.

\section{Eine empirische Bestandsaufnahme}

Welche Auswirkungen der Digitalisierung sind bereits heute zu beobachten? Welche weiteren Folgen zeichnen sich schon jetzt ab? Auf Grundlage aktueller Daten und Forschungsergebnisse gibt die folgende kurze Bestandsaufnahme Antworten auf diese Fragen. Es zeigt sich, dass die digitalisierte Arbeitswelt längst keine Utopie mehr ist, sondern schon seit Jahren Einzug in den Alltag von immer mehr Berufsfeldern hält. Insofern ist die Digitalisierung eher als ein langfristig voranschreitender Prozess denn als eine kurzfristig bevorstehende Umwälzung zu verstehen. Ihre Effekte lassen sich dennoch bereits heute klar empirisch belegen - allerdings fallen diese häufig (zumindest bislang) weit weniger dramatisch aus als gemeinhin vermutet.

\subsection{Wandel der Berufe und Berufsbilder}

Bisherige Forschungsergebnisse zu den Auswirkungen der Entwicklungen in der IKT zeigen bereits konkret den Wandel traditioneller Berufe und Berufsbilder. Formale Qualifikationen erscheinen nicht mehr als das entscheidende Kriterium für eine zukunftssichere Karriere. Global betrachtet führt eine zunehmende Automatisierung von Arbeitsprozessen tendenziell zu einer Polarisierung der Arbeitsmärkte und einer Verdrängung von Arbeitnehmern aus dem mittleren Qualifikationsniveau (vgl. etwa Autor et al., 1998; Acemoglu/Autor, 2011).

Es ist zudem absehbar, dass die fortschreitende Integration von Produktionsprozessen und Kommunikationstechnologien im Rahmen einer Industrie 4.0 weitere bedeutende 
Umstrukturierungen nach sich ziehen wird (vgl. Graetz/Michaels, 2015). Von zukünftigen Rationalisierungsmaßnahmen werden wohl vor allem Berufe bedroht sein, die sich durch hohe Anforderungen in den Bereichen Präzision und insbesondere Routine auszeichnen (vgl. Goos et al., 2014). Hier sind Maschinen den Menschen überlegen. Zukunftssichere Beschäftigungsfelder zeichnen sich dagegen wohl vor allem durch hohe Anforderungen in den Bereichen Kreativität, soziale Intelligenz und unternehmerisches Denken aus.

Eine Übertragung der oben erwähnten Studie von Frey/Osborne (2013) auf den deutschen Kontext liefert - zumindest vordergründig - ähnliche Resultate für das Automatisierungspotenzial von Arbeitsplätzen wie in den Vereinigten Staaten (vgl. Bonin et al., 2015). So wird bei einem analogen Vorgehen anhand von Experteneinschätzungen ermittelt, dass der mittelfristig von Maschinen, Robotern und Computerprogrammen bedrohte Anteil der Beschäftigung mit 42\% nur unwesentlich geringer ausfällt als in den USA. Ein alternativer, tätigkeitsbasierter Ansatz zeichnet jedoch ein weniger dramatisches Bild: Demnach weisen nur $12 \%$ der Arbeitsplätze in Deutschland ein relativ hohes Automatisierungsrisiko auf (und 9\% der Arbeitsplätze in den Vereinigten Staaten; vgl. Bonin et al., 2015). ${ }^{2}$ Ähnlich lautet das Ergebnis der Berechnungen von Dengler/Matthes (2015), wonach 15\% der sozialversicherungspflichtig Beschäftigten im Jahr 2013 in einem Beruf arbeiteten, der in hohem Maße durch Computer substituiert werden kann. Auch wenn dieser Prozentsatz in seiner Dimension nicht gering geachtet werden darf, ist doch eine gewisse Vorsicht bei der Beurteilung des technischen Automatisierungspotenzials angemessen - seine negativen Arbeitsmarkteffekte werden bisweilen stark überschätzt.

Die Veränderungen in der quantitativen Bedeutung von Berufen in Deutschland folgen unbestreitbar einem langfristigen Trend (vgl. auch Eichhorst/Buhlmann, 2015; Eichhorst et al., 2015). So zeigt Abbildung 1, dass sich das Wachstum der Erwerbstätigkeit im Zeitraum von 1996 bis 2011 auf vergleichsweise wenige Berufsgruppen konzentriert hat. Zu den Berufsgruppen mit einer relativ starken Zuwachsrate von mehr als 20\% gehören die folgenden Berufsgruppen: personenbezogene Dienstleistungsberufe und Sicherheitsbedienstete (in Abbildung 1 die Berufsgruppe 51), Hilfsarbeitskräfte im Verkauf und bei Dienstleistungen (91), biowissenschaftliche und Gesundheitsfachkräfte (32), nicht-wissenschaftliche Lehrkräfte (33), sonstige Wissenschaftler (24), sonstige Fachkräfte mittlerer Qualifikation (34), Geschäftsleiter (12), Physiker, Mathematiker und Ingenieurwissenschaftler (21) sowie Biowissenschaftler und Mediziner (22). Umgekehrt weisen im selben Beobachtungszeitraum folgende Berufsgruppen

\footnotetext{
${ }^{2}$ Ähnliche Ergebnisse liefert eine Analyse für 21 OECD-Länder (vgl. Arntz et al., 2016). Demnach sind im Schnitt (nur) 9\% der Jobs in diesen Ländern nach einem tätigkeitsbasierten Ansatz automatisierbar. Allerdings existieren relativ große Unterschiede zwischen den einzelnen Ländern. So führen Deutschland und Österreich die Liste mit einem Automatisierungsrisiko von $12 \%$ an, während dieser Wert in Korea, Estland oder Finnland nur etwa halb so hoch ausfällt.
} 
eine vergleichsweise starke negative Wachstumsrate von 10\% und mehr auf: Hilfskräfte in der Landwirtschaft und Fischerei (92), Fachkräfte in der Landwirtschaft und Fischerei (61), diverse handwerkliche Berufsgruppen $(71,73,74)$, Bediener stationärer und verwandter Anlagen (81) sowie angehörige gesetzgebender Körperschaften und leitende Verwaltungsbedienstete (11). In diesen Zahlen kommt einerseits der fortschreitende Strukturwandel von landwirtschaftlichen und handwerklichen Berufen hin zu Berufen im tertiären Sektor zum Ausdruck. Deutschlands Wandel zu einer Dienstleistungsgesellschaft hat sich in dem betrachteten Zeitraum dynamisch fortgesetzt. Andererseits wird deutlich, dass gleichzeitig vor allem Berufsgruppen mit bestimmten Anforderungen - hauptsächlich im tertiären Sektor erheblich an Bedeutung gewonnen haben. Zu diesen Anforderungen zählen wie bereits erwähnt Kreativität (z.B. Wissenschaftler), soziale Intelligenz (z.B. Lehrer) und unternehmerisches Denken (z.B. Geschäftsleiter).

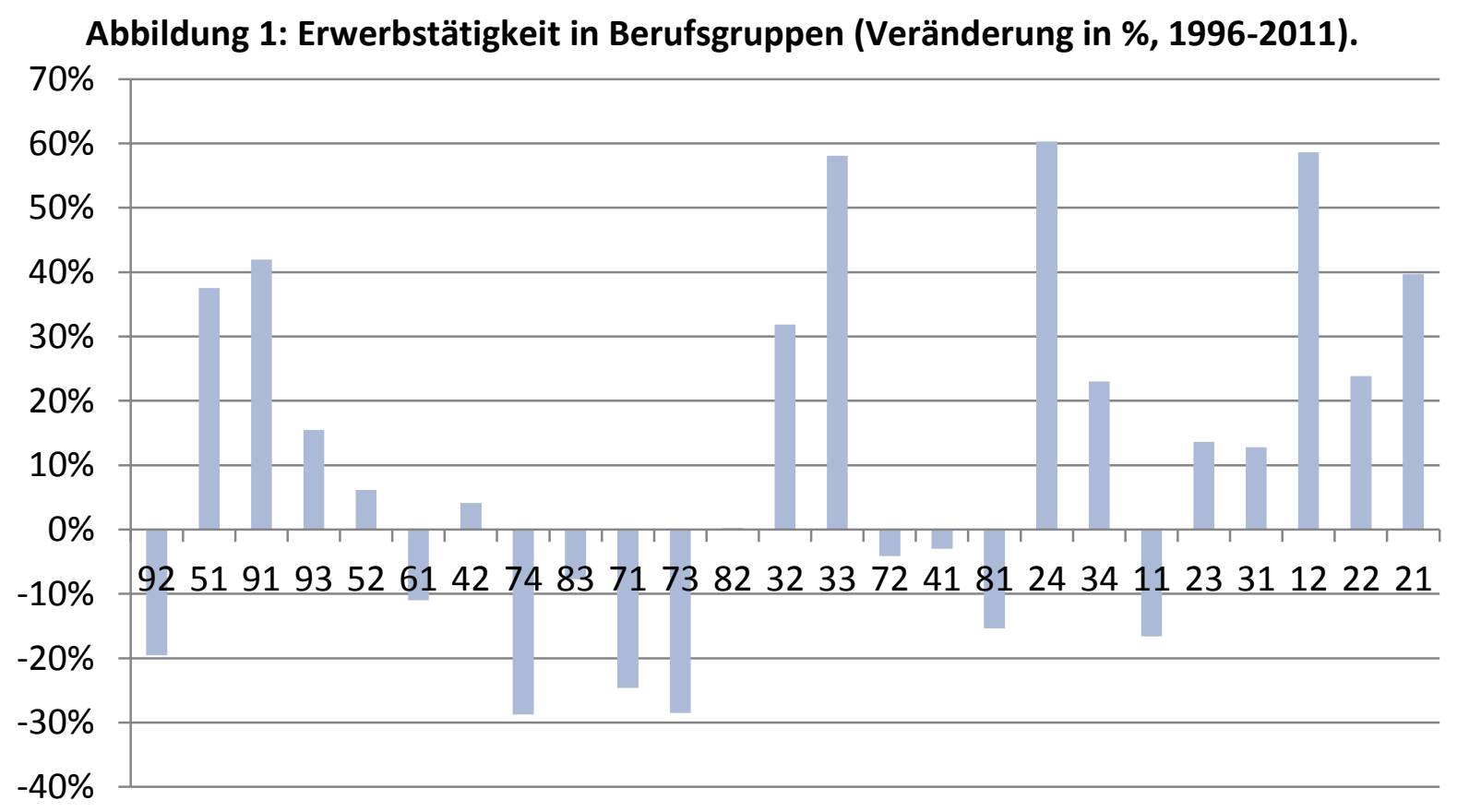

Quelle: Mikrozensus, eigene Berechnungen; Berufsgruppen nach ISCO-88 (vgl. Tabelle im Anhang) sortiert nach Medianlöhnen 2010 aus SIAB.

Wie Abbildung 2 veranschaulicht, gehen diese Entwicklungen in der Tat mit einer gewissen Tendenz zur Polarisierung des deutschen Arbeitsmarktes einher, durch die Arbeitnehmer aus dem mittleren Lohn- und Qualifikationssegment tendenziell verdrängt werden. Allerdings ist diese Entwicklung in der Vergangenheit in Deutschland weniger dramatisch verlaufen als in anderen europäischen Ländern (vgl. Goos et al., 2014; Eurofound, 2015). Gleichwohl ist die Beschäftigungsentwicklung zwischen 1995 und 2010 für Berufsgruppen am oberen und unteren Ende der Bruttolohnverteilung stärker positiv ausgefallen, während Berufsgruppen mit einem mittleren Lohnniveau ein eher schwaches Beschäftigungswachstum zu verzeichnen 
hatten. Exemplarisch für diese Entwicklungen stehen auf der einen Seite die Berufsgruppen der Wissenschaftler (in Abbildung 2 die Berufsgruppen 21 und 22) mit einem hohen Lohnniveau und einem sehr hohem Beschäftigungswachstum, auf der anderen Seite handwerkliche Berufsgruppen (71 und 74) mit mittlerem Lohnniveau und einem recht starken Rückgang der Beschäftigung im Beobachtungszeitraum. Demgegenüber hatte die Berufsgruppe der landwirtschaftlichen Hilfsarbeitskräfte (92), obgleich sie am unteren Ende der Bruttolohnverteilung positioniert ist, ebenfalls einen vergleichsweise starken Beschäftigungszuwachs zu verzeichnen.

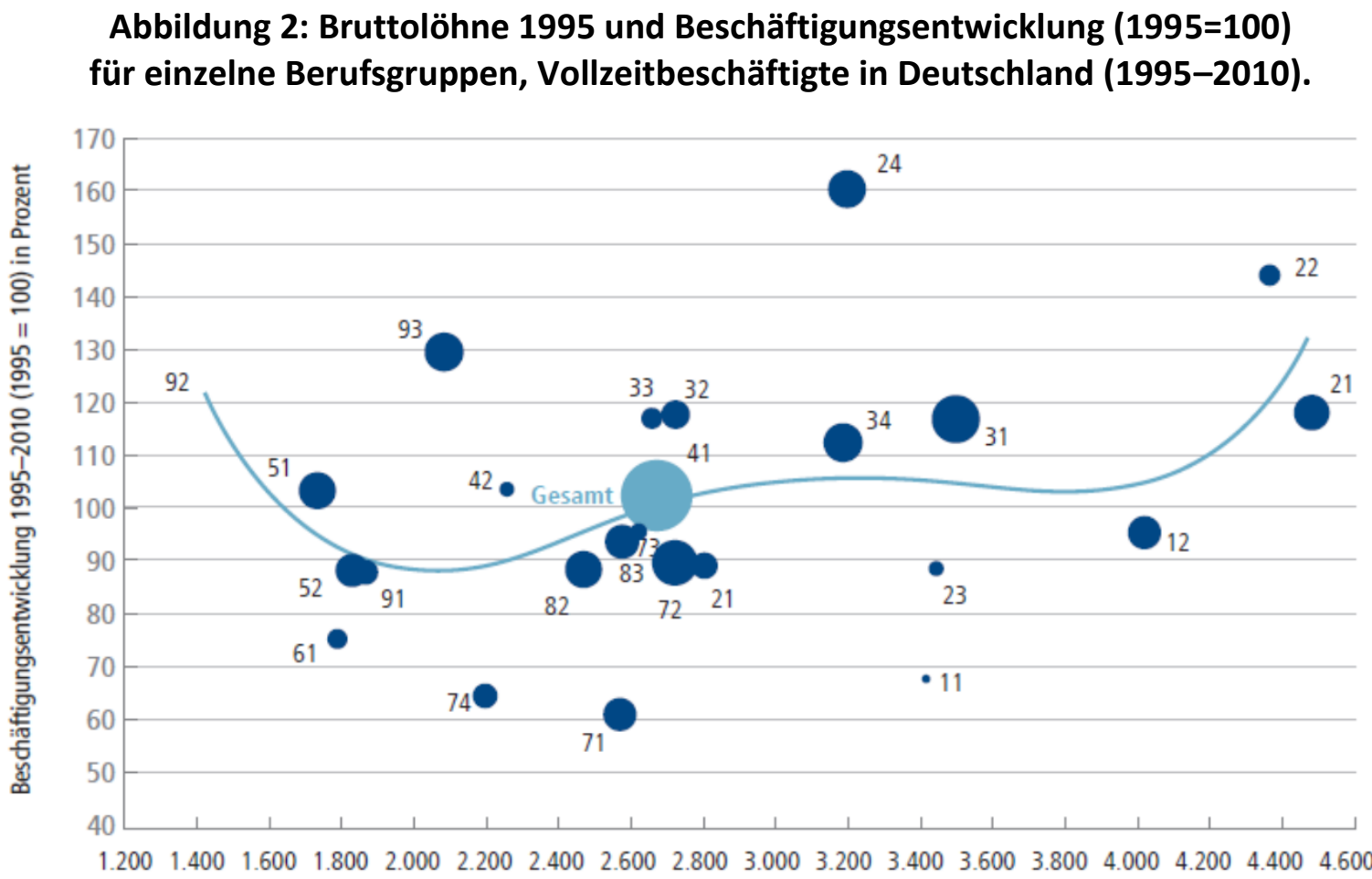

Quelle: SIAB, eigene Berechnungen; Berufsgruppen nach ISCO-88 (vgl. Tabelle im Anhang).

Insofern lässt sich konstatieren, dass sich wichtige Befunde und Prognosen der internationalen Literatur auch zu einem gewissen Grad in deutschen Daten widerspiegeln. Deutlich wird, dass vor allem kreative Berufe, Berufe in der Unternehmensleitung und -beratung sowie Gesundheits-, Sozial- und Erziehungsberufe in den letzten 20 Jahre starke Wachstumsraten der Beschäftigung zu verzeichnen hatten. Bei einer gleichzeitigen, moderaten Polarisierung ergibt sich damit das Bild eines Arbeitsmarktes, der durch eine tendenziell rückläufige Bedeutung von Routinetätigkeiten insbesondere im mittleren Lohn- und Qualifikationsniveau gekennzeichnet ist.

Allerdings ist an dieser Stelle ebenfalls festzuhalten, dass bislang insgesamt kein Einbruch der Erwerbstätigkeit festgestellt werden kann - weder in der Gesamtzahl der Erwerbstätigen noch 
im Arbeitsvolumen. ${ }^{3}$ Bezahlte Erwerbsarbeit ist also entgegen manchen Vorhersagen nicht auf dem Rückzug, sondern ihr Ausmaß ist bemerkenswert robust. Insbesondere höher qualifizierte und komplexere, interaktive Tätigkeiten gewinnen an Bedeutung im Hinblick auf Erwerbstätigkeit und haben zugleich eine positive Entlohnungstendenz.

\subsection{Wandel der Erwerbsformen}

Unsere Arbeitswelt ist mehr denn je in Bewegung; sie wird immer vielschichtiger und auch flexibler. Dies hat nicht nur Auswirkungen auf Berufe und Berufsbilder, sondern auch auf unsere Erwerbs- und Arbeitsformen (vgl. Rinne/Zimmermann, 2016). So zählen neben der verstärkten Projektarbeit auch das vernetzte Arbeiten, Denken und Handeln zu den bestimmenden Merkmalen der Erwerbsgesellschaft von morgen. Deshalb werden flexible Arbeitszeitmodelle mit Gleitzeit, Arbeitszeitguthaben, Heimarbeit und variablen Zeitplanungen eher zum Standard als zur Ausnahme. Die zunehmende Knappheit des Angebots an (qualifizierten) Arbeitskräften bei gleichzeitig wachsendem Wettbewerbs- und Innovationsdruck wird weitere Innovationen bei den Arbeitsstrukturen nach sich ziehen. Flexibilität in der Einteilung der Arbeitszeit oder bei der Wahl des Arbeitsortes wird auch die Grenze zwischen Privat- und Arbeitsleben in ihrer bislang bekannten Form noch weiter auflösen - mit wünschenswerten Effekten wie einer besseren Vereinbarkeit von Familie und Beruf, aber auch mit möglichen negativen Auswirkungen (vgl. etwa Eichhorst et al., 2016).

Im Ergebnis wird das tradierte gesellschaftspolitische Leitbild des „Normalarbeitsverhältnisses" im Sinne eines unbefristeten Vollzeitarbeitsplatzes immer stärker von anderen Erwerbsformen ergänzt. Wie Abbildung 3 zeigt, resultiert dies allerdings weniger aus einem absoluten Rückgang des Anteils von Normalarbeitsverhältnissen. Zwar ist dieser Anteil im Beobachtungszeitraum um insgesamt 5 Prozentpunkte zurückgegangen (von 45\% im Jahr 1992 auf 40\% im Jahr 2014), doch lag dieser Wert bereits seit Mitte der 1990er Jahre nahezu konstant bei $40 \%$. Bemerkenswert ist vielmehr der recht deutliche Rückgang des Anteils der inaktiven erwerbsfähigen Bevölkerung um 8 Prozentpunkte (von 26\% im Jahr 1992 auf 18\% im Jahr 2014) sowie der gleichzeitige Anstieg der Bedeutung anderer, nach herkömmlicher Lesart „atypischer“ Erwerbsformen wie etwa Teilzeittätigkeiten, befristete Tätigkeiten, Zeitarbeit und Minijobs. Der Anteil der Arbeitslosen ist dagegen im Beobachtungszeitraum mit Werten von $6 \%$ bis $8 \%$ relativ konstant geblieben.

\footnotetext{
${ }^{3}$ So erreichte die Zahl der Erwerbstätigen in Deutschland im Jahr 2015 einen neuen Höchststand seit der Wiedervereinigung mit durchschnittlich rund 43 Millionen Personen (Statistisches Bundesamt, 2016). Das Arbeitsvolumen der in Deutschland beschäftigten Arbeitnehmer stieg zuletzt ebenfalls auf knapp 50 Milliarden Stunden an und hat damit wieder ein Niveau erreicht, welches zuletzt zu Beginn der 1990er Jahre gemessen wurde (IAB, 2015).
} 
Abbildung 3: Erwerbsfähige Bevölkerung nach Haupterwerbsstatus (1992-2014).

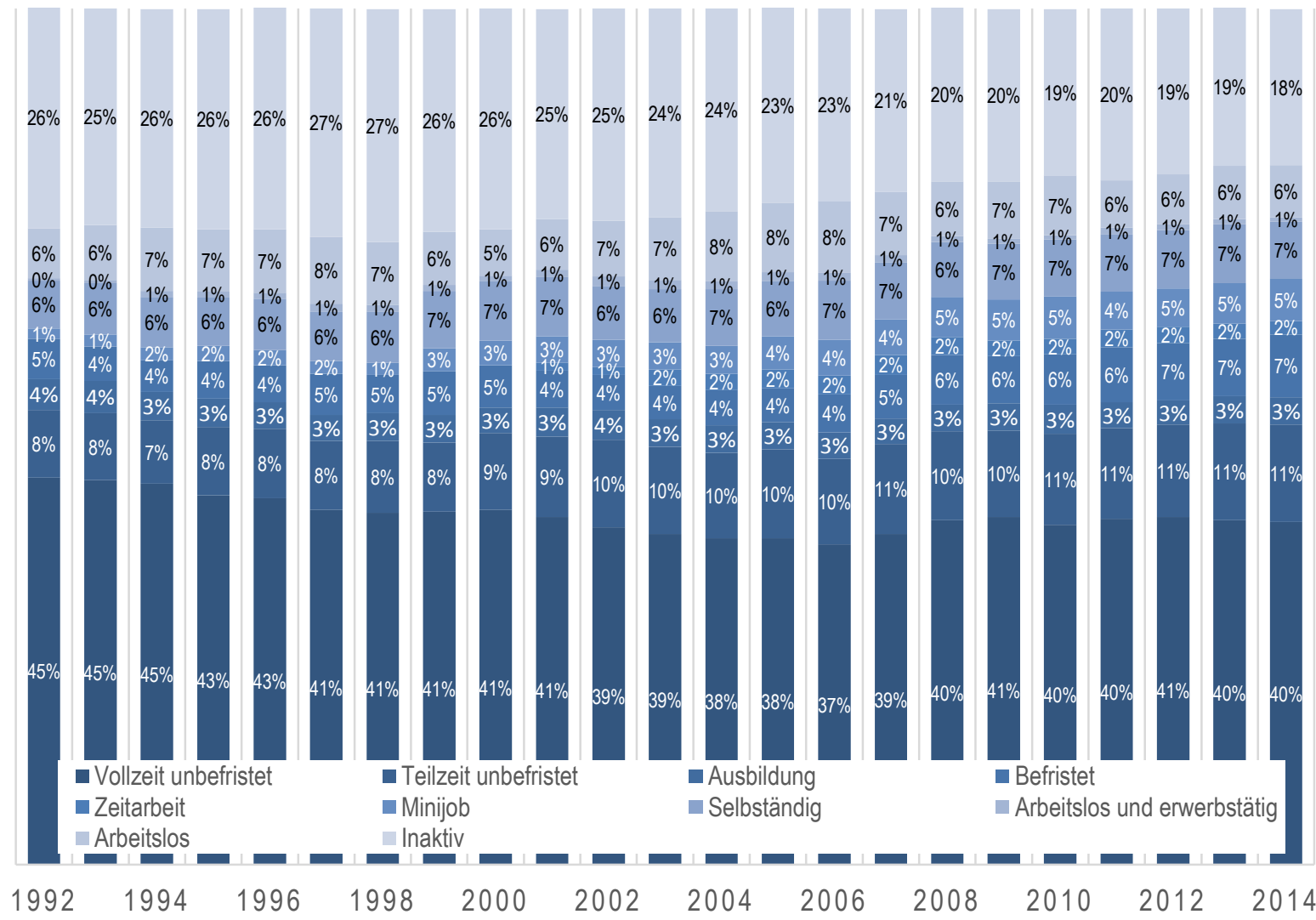

Quelle: Eichhorst et al. (2016). Dort anhand von SOEP 1992-2014 (Querschnittsgewichtung für Personen) und eigenen Berechnungen.

Abbildung 4 beleuchtet die Zunahme der atypischen Beschäftigung in verschiedenen Berufsgruppen im Zeitraum von 1996 bis 2011 näher. Eine Zunahme dieser Beschäftigungsformen erfolgte demnach fast durchgängig und in nahezu allen Berufsgruppen. Ein klarer Zusammenhang zum Lohn- oder Qualifikationsniveau lässt sich nicht ausmachen, doch es fällt sowohl die überdurchschnittlich hohe Zunahme in einfacher qualifizierten Segmenten wie auch der besonders hohe Umfang atypischer Beschäftigung in Verkauf und Verwaltung auf (91: Hilfsarbeitskräfte im Verkauf und bei Dienstleistungen, 11: Verwaltungsbedienstete). Von einem ausgeprägten Effekt der Digitalisierung kann hier jedenfalls nicht gesprochen werden.

Wohl aber zeigt sich anhand dieser Daten, wie groß inzwischen in Deutschland die Vielfalt an Erwerbsformen und Flexibilitätsmustern in einzelnen Berufen und Wirtschaftszweigen geworden ist. Flexible oder atypische Beschäftigungsverhältnisse sind bislang unverändert vor allem in Bereichen anzutreffen, in denen Arbeitskräfte leicht verfügbar bzw. ersetzbar sind, aber längst auch darüber hinaus fester Bestandteil unserer Arbeitswelt. Da dieser Wandel allerdings schon vor mehr als zwei Jahrzehnten eingesetzt hat und auch stark von institutionellen Reformen beeinflusst wurde, spielen hier Digitalisierungseffekte allenfalls eine untergeordnete Rolle. 
Abbildung 4: Anteil der atypischen Beschäftigung (Minijob, Befristung, Zeitarbeit, Selbständigkeit; 1996-2011).

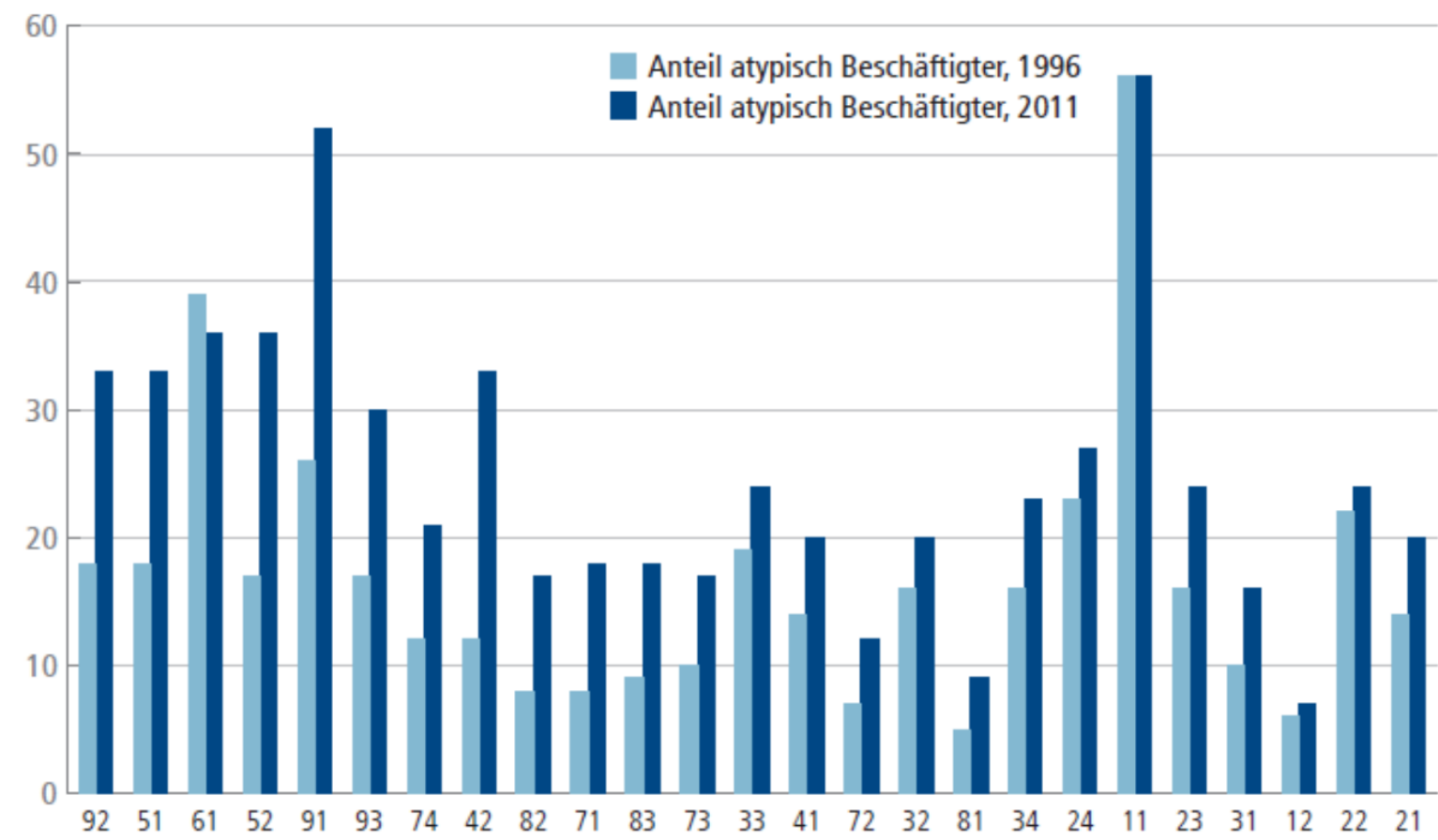

Quelle: Mikrozensus, eigene Berechnungen; Berufsgruppen nach ISCO-88 (vgl. Tabelle im Anhang) sortiert nach Medianlöhnen 2010 aus SIAB.

Umgekehrt scheint die Bereitschaft zu einer auf Dauer angelegten Beschäftigung stark vom jeweiligen betrieblichen Produktionsmodell und von der Verfügbarkeit spezifischer Qualifikationen abhängig zu sein. Im Ergebnis ist der Anteil von Normalarbeitsverhältnissen zumindest seit der Jahrtausendwende bemerkenswert stabil geblieben. Neben einer Zunahme der atypischen Beschäftigung ist als herausragende Entwicklung auf dem deutschen Arbeitsmarkt daher nicht etwa ein Rückzug des tradierten gesellschaftspolitischen Leitbildes zu nennen. Wirklich beachtlich ist wohl eher die Aktivierung eines erheblichen Anteils der erwerbsfähigen Bevölkerung durch neue Flexibilisierungsangebote: Die Zahl der inaktiven Personen ist in den letzten 20 Jahren stark zurückgegangen (vgl. dazu auch Arnold et al., 2016).

\subsection{Rolle der Plattform-Ökonomie}

Es zeichnet sich ein weiterer Wandel mit möglicherweise bedeutenden Auswirkungen ab. So sprechen verschiedene Indizien für eine größere Rolle der sogenannten Plattform-Ökonomie. Das Beispiel der Firma Uber zeigt, wie auf einem „virtuellen Marktplatz" Gelegenheitsfahrer und Fahrgäste zusammengebracht werden und so das Taxi-Gewerbe erheblich unter Druck gesetzt wird (vgl. für die Vereinigten Staaten etwa Hall/Krueger, 2015). Das grundlegende Prinzip lässt sich zudem auf viele andere Branchen übertragen - einschließlich solcher, in denen vorwiegend Fach- und Wissensarbeiter tätig sind. Entsprechende Plattformen 
existieren auch bereits: Hier werden zum Beispiel Aufträge für Werbetexter, Programmierer oder Designer einzeln ausgeschrieben und auch abgewickelt. Interessierte Unternehmen richten gewissermaßen einen Aufruf an eine "Crowd" von mehr oder minder professionell via Plattformen organsierten Anbietern und aquirieren auf diese Weise Zulieferdienste, für die sie im Betrieb keine Ressourcen an Humankapital oder Maschinen mehr vorhalten müssen (vgl. zum Phänomen des Crowdsourcing u.a. Leimeister/Zogaj, 2013; Leimeister et al., 2016). Für die Anbieter der entsprechenden Dienstleistungen bietet sich auf diese Weise vielfach die gern genutzte Gelegenheit, ihre Arbeitsleistung in einem Format anzubieten, das ihren Bedürfnissen entspricht. Andere unterwerfen sich notgedrungen solchen Regularien, um mit ihrem Angebot weiter auf dem Markt bestehen zu können. In jedem Fall sorgt die steigende Nachfrage von Unternehmen nach Crowdworkern dafür, dass mehr Privatpersonen über diesen digitalen Weg ihre Dienstleistungen anbieten und damit klassischen Geschäftsmodellen Konkurrenz machen. Zugleich gerät das Preisgefüge für diese Dienstleistungen tendenziell unter Druck.

Darüber hinaus erfolgt auch eine Verlagerung von Risiken der Arbeitgeber auf die Beschäftigten, genauer gesagt auf die meist (formal) selbstständigen Auftragnehmer (vgl. Eichhorst/Spermann, 2015, 2016). Denn Plattformen wie Uber verstehen sich nicht als Arbeitgeber, sondern lediglich als Vermittler. Beschäftigte der Plattform-Anbieter sind mithin keine klassischen Arbeitnehmer, sondern Selbstständige, die sämtliche Risiken - zum Beispiel bei Unfall oder Krankheit - sowie Leistungen für Altersvorsorge, Arbeitslosigkeit oder Pflege selbst tragen müssen. Damit ergeben sich neue Herausforderungen für die sozialen Sicherungssysteme.

Eine Verlagerung unternehmerischer Risiken auf Arbeitnehmer ist jedoch auch innerhalb von Unternehmen zu beobachten. An die Stelle von Handlungsanweisungen treten zunehmend Zielvereinbarungen im Rahmen von Projekten oder projektartiger Aufgabenstellungen, die weitgehend eigenverantwortlich umzusetzen sind; strenge Hierarchien lösen sich auf und erfolgsabhängige Entlohnungen gewinnen an Bedeutung (Schneider, 2011). Arbeitnehmer werden auf diese Weise zu Unternehmern im Unternehmen. Dementsprechend wird auch unternehmerisches Denken immer mehr zu einer Schlüsselkompetenz in der Arbeitswelt von abhängig Beschäftigten.

Welches Ausmaß hat die Plattform-Ökonomie heute in Deutschland? Eine genaue Antwort auf diese Frage fällt zugegebenermaßen schwer, auch weil die Verfügbarkeit geeigneter Daten der tatsächlichen Entwicklung und Realität und auf dem Arbeitsmarkt mit einiger zeitlicher Verzögerung folgt und bestimmte Phänomene kaum angemessen abgebildet werden. Abbildung 5 stellt den Versuch dar, das Ausmaß der Plattform-Ökonomie mithilfe von SoloSelbstständigkeit und Selbstständigkeit zu messen. Demnach war bei den Solo- 
Selbstständigen ein starker Anstieg von Mitte der 1990er bis etwa Mitte der 2000er Jahre zu verzeichnen, insbesondere bei Qualifizierten, Frauen und Teilzeitkräften. Hingegen sind die Veränderungen der Anzahl und des Anteils von Selbständigen vergleichsweise unauffällig.

Abbildung 5: Entwicklung der Zahl der Solo-Selbständigen, der Selbständigen mit Beschäftigten und Anteil der Selbständigen an allen Erwerbstätigen (1991-2014).

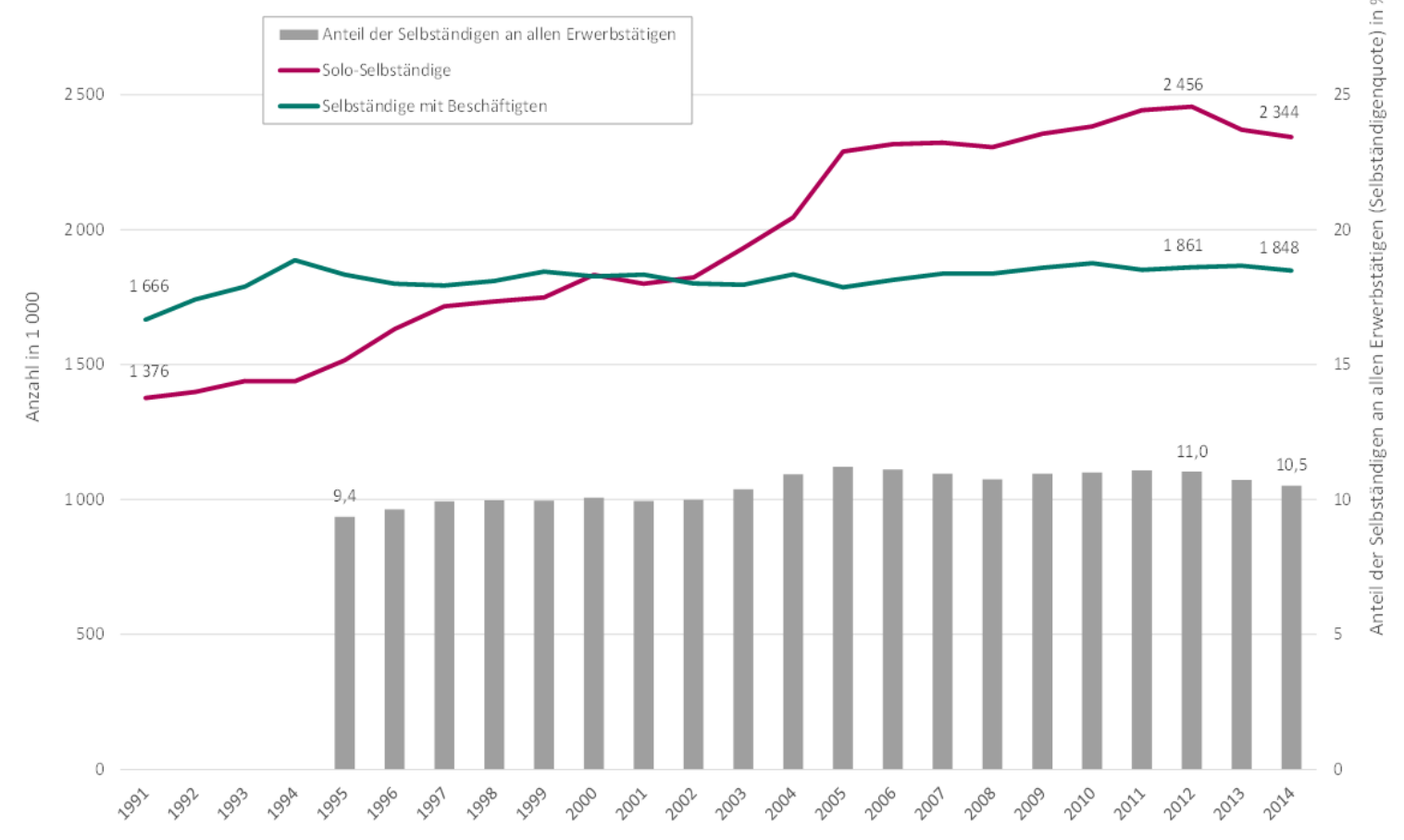

Quelle: Brenke/Beznoska (2016). Dort unter Verwendung von Eurostat-Daten.

Darüber hinaus können im Zeitraum zwischen 2002 und 2005 viele Neugründungen in Form von Solo-Selbstständigkeit durch die Förderung der „Ich-AG" und durch die Lockerung des Handwerksrechts im Jahr 2004 erklärt werden. Neben der kaum identifizierbaren Rolle der Plattform-Ökonomie in den letzten Jahren kommen in den betrachteten Zahlen daher auch die Auswirkungen von geänderten institutionellen und rechtlichen Rahmenbedingungen zum Ausdruck. Umgekehrt ist jedoch auch ein Rückgang der Zahl der Solo-Selbständigen seit dem Jahr 2012 zu verzeichnen - übrigens auch bei denjenigen, die nicht von der Bundesagentur für Arbeit gefördert werden. Im Ergebnis lassen sich somit - zumindest bislang - keine Hinweise auf ein (massives) Anwachsen der Solo-Selbstständigkeit anhand der vorliegenden Daten nachweisen. Der bisherige, eher überschaubare Anstieg scheint eher mit anderen Faktoren als mit einem Anwachsen der Plattform-Ökonomie zusammenzuhängen und wirkt derzeit nicht dramatisch. 
Studien zur Einkommenssituation von Solo-Selbstständigen weisen jedoch auf eine vergleichsweise hohe Spreizung der Verdienste und eine nur gering ausgeprägte Fähigkeit oder Bereitschaft zur Altersvorsorge hin (vgl. etwa Brenke 2013, 2015; Brenke/Beznoska, 2016). Eine ähnliche Entwicklung lässt sich im Übrigen bei den Erwerbstätigen beobachten, die in repräsentativen Befragungen wie dem SOEP angeben, gelegentlich oder regelmäßig eine Nebentätigkeit auszuüben. Seit den frühen 2000er Jahren gehen nach unseren Auswertungen des SOEP stabil etwa 2,5 bis 3,0\% der Erwerbsfähigen gelegentlich oder regelmäßig einer Nebentätigkeit nach. Hier werden jedoch vermutlich eher abhängige Beschäftigungsverhältnisse angegeben, d.h. Minijobs, deren Verbreitung nach ihrer Reform seit dem Jahr 2003 zugenommen hat.

Bislang steht die Plattform-Ökonomie (als eine Form der Sharing Economy) erst am Anfang ihrer Entwicklung. Alle Anzeichen deuten darauf hin, dass ihre tatsächliche Bedeutung nicht nur in Deutschland bislang noch sehr überschaubar ist. So wird selbst in den USA, die in dieser Hinsicht eine Vorreiterrolle einnehmen, der Anteil der Erwerbstätigen, die ihre Dienstleistungen über Online-Plattformen wie Uber oder TaskRabbit anbieten, zuletzt auf lediglich 0,4\% bis 0,5\% geschätzt (vgl. Katz/Kruger, 2016; Farrell/Greig, 2016; Harris/Krueger, 2015). Gleichzeitig weisen Befragungen von so genannten „Crowdworkern“ darauf hin, dass in den allermeisten Fällen lediglich Nebentätigkeiten ausgeübt werden, wobei die hieraus generierten Einkünfte andere Einkommensarten im Haushalt ergänzen. Als zusätzlicher Nutzen wird der Austausch im Netzwerk vergleichbar qualifizierter und interessierter virtueller „Kollegen“, der Kontakt zu Unternehmen und der Zugewinn an Erfahrung durch die Crowd-Projektarbeit angesehen (Leimeister/Zogaj, 2013).

Es ist aber davon auszugehen, dass gerade dieses neue Arbeitsmarktsegment eine sehr dynamische Entwicklung nehmen und sich zusehends auch auf höherwertigere Dienstleistungen erstrecken wird. Aufgabe der Sozialpolitik wird es sein, sich frühzeitig genug auf die damit einhergehenden Herausforderungen einzustellen.

\section{Sozialpolitische Herausforderungen}

Wenngleich sich derzeit noch kein massives Wachstum der Solo-Selbstständigkeit beobachten lässt, stellen sich potenziell sozialpolitische Herausforderungen, auf die rechtzeitig Antworten gefunden werden müssen. Hierbei sind zwei Hauptmotive zu beachten: Zum einen fehlt es vielen (Solo)Selbstständigen an einer angemessenen Alterssicherung, so dass sie im Fall einer auf längere Zeit ausgeübten selbstständigen Tätigkeit nicht mit existenzsichernden Renten rechnen können. Damit würden sie auf die steuerfinanzierte Grundsicherung verwiesen. Eine angemessene Vorsorge erscheint vor diesem Hintergrund notwendig. Zum anderen besteht 
eine potenzielle Konkurrenzsituation zwischen Unternehmen mit regulär Beschäftigten, für die auch Arbeitgeberbeiträge zur Sozialversicherung abgeführt werden, und Plattformen, die formal Selbstständige einsetzen und dabei die soziale Absicherung auf diese Personen abwälzen, aber sich selbst nicht als Arbeitgeber sehen. Damit kann eine denkbare Ausweitung formal selbstständiger Tätigkeiten das soziale Absicherungsmodell prinzipiell unterminieren.

Ein erster Ansatzpunkt ist die Abgrenzung zwischen abhängiger und selbstständiger Tätigkeit. Zweifellos verschwimmen die Grenzen zwischen den Erwerbsformen zunehmend. Das impliziert, dass traditionelle Klassifikationen und Schemata nicht mehr zutreffend sind. Eine klare Grenze zwischen abhängig Beschäftigten und Selbstständigen zu ziehen, fällt immer öfter schwer. Im US-amerikanischen Raum, aber auch in Deutschland, wird gleichwohl aufgrund der sozialpolitischen Implikationen über die Abgrenzung von Solo- oder Scheinselbstständigen und abhängig Beschäftigten und damit die Rolle der Arbeitgeber diskutiert. In vielen Fällen gibt es Anzeichen für eine Arbeitgeberfunktion der Plattformen für die von ihnen ökonomisch abhängigen Dienstleister. Auch deshalb wird z.B. für den USamerikanischen Kontext die Einführung einer neuen (gesetzlichen) Kategorie des „Independent Worker" diskutiert, um insbesondere die sozialen Sicherungssysteme mit den Anforderungen der Plattform-Ökonomie in Einklang zu bringen und in die digitale Arbeitswelt zu überführen (vgl. Harris/Krueger, 2015). Österreich hat das Konstrukt eines „freien Dienstvertrags" eingeführt, um den klassischen Werkverträgen eine auf stundengenauer Entgeltabrechnung basierende und vollwertige Sozialversicherungsabgaben beinhaltende Beschäftigungsform zur Seite zu stellen. Besondere Schwierigkeiten stellen sich jedoch bei Privilegien und Leistungen, die an die Arbeitszeit bzw. an Stundenlöhne geknüpft sind (wie etwa Überstundenregelungen oder Mindestlohnvorgaben), da Erwerbstätige in der PlattformÖkonomie nicht mehr nur für einen „Arbeitgeber" tätig sind.

Eine ähnliche Diskussion wird in Deutschland unter dem Aspekt der Werkverträge und der Scheinselbstständigkeit geführt. Je strikter die Anforderungen an eine „echte“ und legale selbstständige Tätigkeit gefasst werden, umso eher lass sich formal Selbstständige, die jedoch ökonomisch abhängig sind, als abhängig Beschäftigte klassifizieren, für die Arbeitgeber Beiträge zu leisten haben. Es steht jedoch immer die Frage der Kontrolle und Rechtsdurchsetzung im Raum.

Ein zweiter Ansatzpunkt ist die Ausweitung des Kreises der von der Sozialversicherung erfassten Erwerbsformen bzw. Personengruppen. Dies gilt insbesondere für die Absicherung bei Alter und Berufsunfähigkeit, daneben aber auch für den Fall der Arbeitslosigkeit. Etwas mehr als die Hälfte der Solo-Selbständigen verfügt über keine Alterssicherung in Form von Einzahlungen in Renten- oder Lebensversicherung (vgl. Brenke/Beznoska, 2016, S. 53). In Deutschland sind nach dem derzeit geltenden und historisch gewachsenen Recht nur einzelne 
Gruppen von arbeitnehmerähnlichen Selbstständigen nach § 2 SGB VI verpflichtend in die gesetzliche Rentenversicherung einbezogen, z.B. Lehrer und Erzieher, Pflegekräfte und Hebammen, Seelotsen und Küstenschiffer, Hausgewerbetreibende, Handwerker, sowie Selbstständige mit nur einem Auftraggeber. Bei den meisten dieser Gruppen besteht keine Versicherungspflicht, wenn Arbeitnehmer beschäftigt werden. Künstler und Publizisten haben Zugang zur Künstlersozialversicherung, die Landwirte zu einer eigenen Alterssicherung. Andere Gruppen von Selbstständigen, gerade in etablierten Professionen wie etwa Ärzte, Architekten, Steuerberater, Notare oder Anwälte, sind im Rahmen berufsständischer Sicherungssysteme abgesichert, während für andere Gruppen Vorsorge nur auf freiwilliger Basis besteht.

Freiwilliger Natur ist bislang im Grundsatz auch die Absicherung in der Arbeitslosenversicherung. Seit 2003 besteht zwar die wenig bekannte Möglichkeit einer freiwilligen Weiterversicherung in der Arbeitslosenversicherung durch ein sogenanntes „Versicherungspflichtverhältnis auf Antrag“ (§ 28a SGB III). Diese Option besteht aber nur, sofern innerhalb der letzten 24 Monate unmittelbar vor der Aufnahme der selbstständigen Tätigkeit mindestens zwölf Monate eine versicherungspflichtige abhängige Beschäftigung ausgeübt oder Arbeitslosengeld I bezogen worden ist. Wird dann vom versicherten Selbstständigen mehr als zwei Mal Arbeitslosengeld I in Anspruch genommen, ist keine Weiterversicherung mehr möglich, sofern nicht eine neue Grundlage aus einem Versicherungspflichtverhältnis erworben wird.

Vor diesem Hintergrund erscheint es plausibel, wenn nicht gar unvermeidlich, Selbstständige in allen "Spielarten" generell in die Sozialversicherung einzubeziehen und ihnen auch im Falle einer angemessenen und existenzsichernden privaten oder berufsständischen Vorsorge keine pauschale Möglichkeit zum „Opt-out“ zu gewähren. Im Gegenteil sollte auch in diesem Fall bis zu einer zu bestimmenden Beitragsgrenze - ein Mindestmaß an Beiträge zur Sozialversicherung von diesem Personenkreis geleistet werden. Die Beiträge der Selbstständigen selbst wären dann durch obligatorische Beiträge der Auftraggeber bzw. der Vermittler/Plattformen (als Arbeitgeber-Äquivalent) zu ergänzen. Sie könten von diesen unmittelbar entrichtet oder aber von den (Solo-)Selbständigen bei der Abrechnung ihrer Dienstleistungen eingefordert und dann weitergeleitet werden. Als Vorbild bietet sich hier das Modell der Künstlersozialkasse mit einer Abgabepflicht der Verwerter an. Daneben kommt auch ein gewisser Anteil an Steuerfinanzierung in Betracht, wie wir ihn bereits derzeit in der gesetzlichen Rentenversicherung und der Künstlersozialversicherung kennen. Hier würden natürlich auch Steuereinnahmen aus unternehmerischen Tätigkeiten einfließen.

Eine weitere Herausforderung wird sein, in der Plattform-Ökonomie eine Steuer- oder Beitragspflicht zu realisieren. Transaktionen über Plattformen, bei denen ein Auftrag mit 
einem territorialen Bezug zu Deutschland abgewickelt wird - wenn etwa Vermittler, Auftraggeber oder Auftragnehmer in Deutschland ansässig sind - können mit einer entsprechenden „Sozialabgabe“ belastet werden, vergleichbar der Umsatzsteuer. Klar ist, dass dann Anreize zu formal selbstständigen Tätigkeiten verringert werden, weil die Beitragspflicht zu höheren Preisen bzw. Arbeitskosten führen wird. Dies wäre der Preis für einen universellen Einbezug in den Sozialstaat und die Vermeidung eines "unfairen" Wettbewerbs zulasten der Sozialkassen bzw. der Steuerzahler.

Der Vorteil einer universelleren Sozialversicherung liegt sicher darin, dass die Problematik der Abgrenzung von selbstständiger, scheinselbstständiger und abhängiger Beschäftigung, die derzeit auch in Deutschland erneut im Kontext von Werkverträgen diskutiert wird, in ihrer Substanz entschärft wird. Eine Vision für die Zukunft ist dann eine „Bürgerversicherung 4.0" unter gleichberechtigtem, also auch privilegienfreien Einschluss aller Beschäftigten. Ein solches Solidarmodell, dem sich niemand entziehen kann, erhält durch die sich abzeichnenden Beschäftigungstrends und durch den daraus resultierenden veränderten Absicherungsbedarf jedenfalls neue Argumente. ${ }^{4}$

Darüber hinaus bietet die Digitalisierung der Arbeitswelt und der Einzug intelligenter Maschinen in den Arbeitsalltag von immer mehr Beschäftigtengruppen Anlass genug, Modelle der Mitarbeiter-Kapitalbeteiligung neu - auch im Sinne der Altersvorsorge - zu konzipieren und flächendeckender als bisher bereitzustellen. Unterstrichen wird diese Notwendigkeit nicht zuletzt durch die lang anhaltende Phase ausbleibender Renditen klassischer Anlageformen und Altersvorsorgemodule.

\section{Zusammenfassung und Fazit}

Die verbreiteten Spekulationen über die rasante Veränderung unserer Arbeitswelt infolge ungeahnter technologischer Neuerungen sind Ausdruck einer beträchtlichen Ungewissheit über das Ausmaß der Anpassungsnotwendigkeiten in Wirtschaft und Gesellschaft. Die Digitalisierung ist für viele von uns zu einem Synonym für unkontrollierbare technische Entwicklungen in der Arbeitswelt geworden, denen die Individuen schutzlos ausgeliefert sind und die vermeintlich in massive Arbeitsplatzverluste und gravierende soziale Ungleichheit münden werden. Auf der anderen Seite werden oft hochfliegende Erwartungen formuliert, die darauf setzen, dass sich die Erfahrungen mit dem technischen Fortschritt auch künftig unverändert fortsetzen und somit nach einer Übergangsphase von Beschäftigungsabbau und

\footnotetext{
${ }^{4}$ So verfolgt z.B. Österreich in seiner Gesundheitspolitik schon seit geraumer Zeit den Ansatz einer „Bürgerversicherung“ (vgl. etwa Opielka, 2004).
} 
Rationalisierung neue, kreative Arbeitsplätze in hinreichender Zahl entstehen werden und der gesellschaftliche Wohlstand gesichert oder gar weiter ausgebaut werden könne.

Die empirische Bestandsaufnahme im vorliegenden Beitrag zeichnet das Bild eines Arbeitsmarktes, der einen tiefgreifenden Wandel vollzieht. Zumindest in der Vergangenheit waren jedoch viele andere Faktoren (z.B. institutionelle und demografische) dafür sehr prägend und bestimmend. Am Beispiel der vieldiskutierten Plattform-Ökonomie lässt sich zeigen, dass ihr derzeitiges Ausmaß insgesamt noch sehr überschaubar ist. In Deutschland ist die Anzahl der Solo-Selbständigen seit dem Jahr 2012 sogar rückläufig und der Anteil der Erwerbstätigen in der Plattform-Ökonomie ist selbst in den USA noch sehr gering. Außerdem zeigt sich zumindest bislang, dass Digitalisierung nicht Massenarbeitslosigkeit bedeutet. Im Gegenteil: Das Ausmaß der Erwerbstätigkeit war den letzten Jahren in Deutschland bemerkenswert robust - bezogen sowohl auf die Anzahl der Erwerbstätigen als auch auf das Arbeitsvolumen in Stunden.

Doch das Potenzial des durch die Digitalisierung ausgelösten Wandels ist enorm, und einschneidende Entwicklungen stehen uns womöglich noch bevor. Schon in der Vergangenheit konnten (zumindest nach herkömmlichen Maßstäben) „kleinere“ Anbieter ganze Märkte und Branchen unter Druck setzen und auf den Kopf stellen. Insofern ist jetzt die Zeit gekommen, neue sozialpolitische Herausforderungen durch die Digitalisierung zu identifizieren, geeignete Maßnahmen zu ergreifen und entsprechende Rahmenbedingungen zu schaffen. Denn beim Übergang in die digitale Arbeitswelt wird es zweifellos ganz entscheidend darauf ankommen, das Verhältnis von Gewinnern und Verlierern genau im Blick zu behalten. Aus diesem Grund gewinnt auch unter sozialpolitischen Aspekten der Ausbau von Mitarbeiterbeteiligungsformaten weiter an Bedeutung.

Insbesondere jedoch gilt es die Frage zu beantworten, inwieweit das Konzept der Sozialversicherung, welches an einer prinzipiell langfristigen abhängigen Beschäftigung anknüpft, systematisch für andere Erwerbsformen - und letztlich für alle Erwerbsformen geöffnet werden soll. Eine beitragsfinanzierte Absicherung für das Alter, aber auch für Berufsunfähigkeit und Arbeitslosigkeit unabhängig vom formalen Erwerbsstatus würde Sicherungslücken schließen, den Wechsel und Kombinationen von Erwerbsformen erleichtern und den Wettbewerb der Arbeitskosten zwischen abhängig und selbstständig tätigen Personen entschärfen. 


\section{Literaturverzeichnis}

Acemoglu, Daron/David H. Autor (2011): Skills, Tasks and Technologies: Implications for Employment and Earnings. Handbook of Labor Economics, vol. 4, chap. 12, S. 1043-1171. Amsterdam.

Arnold, Michael/Anselm Mattes/Gert G. Wagner (2016): Normale Arbeitsverhältnisse sind weiterhin die Regel. DIW Wochenbericht 19/2016, S. 419-427.

Arntz, Melanie/Terry Gregory/Ulrich Zierahn (2016): The Risk of Automation for Jobs in OECD Countries: A Comparative Analysis. OECD Social, Employment and Migration Working Papers No. 189.

Autor, David H./Lawrence F. Katz/Alan B. Krueger (1998): Computing Inequality: Have Computers Changed The Labor Market? Quarterly Journal of Economics 113(4), S. 1169-1213.

Autor, David H./David Dorn/Gordon H. Hanson (2016): The China Shock: Learning from Labor Market Adjustment to Large Changes in Trade. NBER Working Paper No. 21906.

BMWi (2015): Impulse für die Digitalisierung der deutschen Wirtschaft - Digitale Agenda des BMWi. Berlin: Bundesministerium für Wirtschaft und Energie (BMWi).

Bonin, Holger/Terry Gregory/Ulrich Zierahn (2015): Übertragung der Studie von Frey/Osborne (2013) auf Deutschland. ZEW-Kurzexpertise Nr. 57. Mannheim: Zentrum für Europäische Wirtschaftsforschung (ZEW).

Brenke, Karl (2013): Allein tätige Selbständige: starkes Beschäftigungswachstum, oft nur geringe Einkommen. DIW Wochenbericht 7/2013, S. 3-16.

Brenke, Karl (2015): Selbständige Beschäftigung geht zurück. DIW Wochenbericht 36/2015, S. 790-796.

Brenke, Karl/Martin Beznoska (2016): Solo-Selbständige in Deutschland - Strukturen und Erwerbsverläufe. Kurzexpertise für das BMAS. (http://www.bmas.de/DE/Service/Medien/ Publikationen/Forschungsberichte/Forschungsberichte-Arbeitsmarkt/fb-465-solo-selbstaendige.html; zuletzt aufgerufen am 9.6.2016).

Brynjolfsson, Erik/Andrew McAfee (2011): Race Against the Machine: How the Digital Revolution is Accelerating Innovation, Driving Productivity, and Irreversibly Transforming Employment and the Economy. Lexington.

Brynjolfsson, Erik/McAfee, Andrew (2014): The Second Machine Age: Wie die nächste digitale Revolution unser aller Leben verändern wird. Kulmbach.

Dengler, Katharina/Britta Matthes, Britta (2015): Folgen der Digitalisierung für die Arbeitswelt Substituierbarkeitspotenziale von Berufen in Deutschland. IAB-Forschungsbericht 11/2015.

Eichhorst, Werner/Florian Buhlmann (2015): Die Zukunft der Arbeit und der Wandel der Arbeitswelt, Wirtschaftspolitische Blätter 62(1), S. 131-148.

Eichhorst, Werner/Patrick Arni/Florian Buhlmann/Ingo E. Isphording/Verena Tobsch (2015): Wandel der Beschäftigung: Polarisierungstendenzen auf dem deutschen Arbeitsmarkt. IZA Research Report No. 68.

Eichhorst, Werner/Holger Hinte/Alexander Spermann/Klaus F. Zimmermann (2015): Die neue Beweglichkeit: Die Gewerkschaften in der digitalen Arbeitswelt. IZA Standpunkt Nr. 82.

Eichhorst, Werner/Michael Kendzia/Hilmar Schneider/Florian Buhlmann (2013): Neue Anforderungen durch den Wandel der Arbeitswelt: Kurzexpertise für die Enquete-Kommission "Wachstum, Wohlstand, Lebensqualität" des Deutschen Bundestages. IZA Research Report No. 51.

Eichhorst, Werner/Alexander Spermann (2015): Sharing Economy - Chancen, Risiken und Gestaltungsoptionen für den Arbeitsmarkt. IZA Research Report No. 69.

Eichhorst, Werner/Alexander Spermann (2016): Sharing Economy: Mehr Chancen als Risiken? Wirtschaftsdienst 96(6), S. 433-439.

Eichhorst, Werner/Verena Tobsch (2015): Flexible Arbeitswelten: Eine Bestandsaufnahme. In: Werner Widuckel et al. (Hrsg.): Arbeitskultur 2020. Wiesbaden. 
Eichhorst, Werner/Verena Tobsch/Caroline Wehner (2016): Neue Qualität der Arbeit? Zur Entwicklung von Arbeitskulturen und Fehlzeiten. In: Bernhard Badura et al. (Hrsg.): Fehlzeiten-Report 2015. Berlin (im Erscheinen).

Eurofound (2015): Upgrading or polarisation? Long-term and global shifts in the employment structure: European Jobs Monitor 2015. Publications Office of the European Union, Luxembourg.

Farrell, Diana/Fiona Greig (2016): The Online Platform Economy: What is the growth trajectory? (https://www.jpmorganchase.com/corporate/institute/institute-insights.htm; zuletzt aufgerufen am 14.6.2016).

Freeman, Richard B. (2015): Who owns the robots rules the world. IZA World of Labor 2015:5.

Frey, Carl Benedikt/Michael A. Osborne (2013): The Future of Employment: How Susceptible are Jobs to Computerisation?, University of Oxford (http://www.oxfordmartin.ox.ac.uk/downloads/ academic/The_Future_of_Employment.pdf; zuletzt abgerufen am 3.6.2016).

Goos, Maarten/Alan Manning/Anna Salomons (2014): Explaining Job Polarization: Routine-Biased Technological Change and Offshoring. American Economic Review 104(8), S. 2509-2526.

Graetz, Georg/Guy Michaels (2015): Robots at Work. IZA Discussion Paper No. 8938.

Hall, Jonathan V./Alan B. Krueger (2015): An Analysis of the Labor Market for Uber's Driver-Partners in the United States. Working Papers No. 587, Princeton University, Industrial Relations Section.

Harris, Seth/Alan B. Krueger (2015): A Proposal for Modernizing Labor Laws for Twenty-First-Century Work: The "Independent Worker". The Hamilton Project, Discussion Paper 2015-10.

Hirsch-Kreinsen, Hartmut (2016): Arbeit und Technik bei Industrie 4.0. Aus Politik und Zeitgeschichte (APUZ) 66(18-19), S. 10-17.

Hobsbawm, Eric J. (1952): The Machine Breakers. Past \& Present 1, S. 57-70.

IAB (2015), Anhangtabellen zu IAB-Kurzbericht 4/2015, 19.2 .2015 (http://doku.iab.de/kurzber/ 2015/kb0415_Anhang.pdf; zuletzt aufgerufen am 6.6.2016).

Katz, Lawrence F./Alan B. Krueger (2016): The Rise and Nature of Alternative Work Arrangements in the United States, 1995-2015

(http://scholar.harvard.edu/files/lkatz/files/katz_krueger_cws_v3.pdf; zuletzt aufgerufen am 14.6.2016).

Keynes, John M. (1930): Economic Possibilities for our Grandchildren. (http://www.econ.yale.edu/ smith/econ116a/keynes1.pdf; zuletzt aufgerufen am 3.6.2016).

Leimeister, Jan M./Shkodran Zogaj (2013): Neue Arbeitsorganisation durch Crowdsourcing - Eine Literaturstudie. Hans Böckler Stiftung - Arbeit und Soziales, Arbeitspapier 287. Düsseldorf.

Leimeister, Jan M./Shkodran Zogaj/David Durward/Ulrich Bretschneider (2015): Neue Geschäftsfelder durch Crowdsourcing: Crowd-basierte Start-ups als Arbeitsmodell der Zukunft. In: Reiner Hoffmann/Claudia Bogedan (Hrsg.) Arbeit der Zukunft - Möglichkeiten nutzen - Grenzen setzen. Frankfurt/M., S. 141-158.

Opielka, Michael (2004). Sozialpolitische Entscheidungen in der Gesundheitspolitik - Reflexionen zu Bürgerversicherung und Gesundheitsprämie. WSI Mitteilungen 1/2004, S. 3-10.

Rifkin, Jeremy (1995): Das Ende der Arbeit. Frankfurt, New York.

Rinne, Ulf/Klaus F. Zimmermann (2016): Die digitale Arbeitswelt von heute und morgen. Aus Politik und Zeitgeschichte (APuZ) 66(18-19), S. 3-9.

Roland Berger (2015): Die Digitale Transformation der Industrie - Was sie bedeutet. Wer gewinnt. Was jetzt zu tun ist. Roland Berger Strategy Consultants, Studie im Auftrag des Bundesverband der Deutschen Industrie e.V. München/Berlin (o.O.) (http://bdi.eu/media/presse/publikationen/ information-und-telekommunikation/Digitale_Transformation.pdf; zuletzt aufgerufen am 24.6.2016).

Schneider, Hilmar (2011): „Fach“-Kräfte für die Arbeit der Zukunft. IZA Standpunkte Nr. 41.

Statistisches Bundesamt (2016): Zahl der Erwerbstätigen stieg im Jahr 2015 auf 43 Millionen Personen. Pressemitteilung Nr. 001 vom 4.1.2016. 


\section{Anhang - Berufsklassifikation nach ISCO-88}

1 Angehörige gesetzgebender Körperschaften, leitende Verwaltungsbedienstete und Führungskräfte in der Privatwirtschaft

11 Angehörige gesetzgebender Körperschaften und leitende Verwaltungsbedienstete

12 Geschäftsleiter

13 Betriebsleiter

\section{Wissenschaftler}

21 Physiker, Mathematiker und Ingenieurwissenschaftler

22 Biowissenschaftler und Mediziner

23 Wissenschaftliche Lehrkräfte

24 Sonstige Wissenschaftler

3 Techniker und gleichrangige nichttechnische Berufe

31 Fachkräfte in der Physik und den Ingenieurwissenschaften

32 Biowissenschaftliche und Gesundheitsfachkräfte

33 Nicht-wissenschaftliche Lehrkräfte

34 Sonstige Fachkräfte (mittlere Qualifikationsebene)

4 Bürokräfte, kaufmännische Angestellte

41 Büroangestellte ohne Kundenkontakt

42 Büroangestellte mit Kundenkontakt

5 Dienstleistungsberufe, Verkäufer in Geschäften und auf Märkten

51 Personenbezogene Dienstleistungsberufe und Sicherheitsbedienstete

52 Modelle, Verkäufer und Vorführer

6 Fachkräfte in der Landwirtschaft und Fischerei

61 Fachkräfte in der Landwirtschaft und Fischerei

62 Arbeitskräfte in der Landwirtschaft und Fischerei (Eigenbedarfsproduktion)

7 Handwerks- und verwandte Berufe

71 Mineralgewinnungs- und Bauberufe

72 Metallarbeiter, Mechaniker und verwandte Berufe

73 Präzisionsarbeiter, Kunsthandwerker, Drucker und verwandte Berufe

74 Sonstige Handwerks- und verwandte Berufe

8 Anlagen- und Maschinenbediener sowie Montierer

81 Bediener stationärer und verwandter Anlagen

82 Maschinenbediener und Montierer

83 Fahrzeugführer und Bediener mobiler Anlagen

9 Hilfsarbeitskräfte

91 Verkaufs- und Dienstleistungskräfte

92 Landwirtschaftliche, Fischerei- und verwandte Hilfsarbeiter

93 Hilfsarbeiter im Bergbau, Baugewerbe, verarbeitenden Gewerbe und Transportwesen

0 Streitkräfte

01 Streitkräfte

Quelle: http://www.gesis.org/fileadmin/upload/dienstleistung/methoden/spezielle_dienste/inhaltsanalyse_berufsklass/isco88_1_.pdf (zuletzt aufgerufen am 9.6.2016) 